\title{
Correction of Left Ventricle Strain Signals Estimated from Tagged MR Images
}

\author{
Mina E. Khalil ${ }^{1}$, Ahmed S. Fahmy ${ }^{1}$, and Nael F. Osman ${ }^{1,2}$ \\ ${ }^{1}$ Center for Informatics Science, Nile University, Cairo, Egypt \\ 2 Johns Hopkins University, School of Medicine, Baltimore, Maryland, USA
}

\begin{abstract}
Strain measurement is a quantity used for assessing the regional function of the left ventricular (LV) of the heart. They are computed by tracking the motion of the non-invasive, virtual tags in the cardiac muscle with time. Tracking these tags gives information for each region of the cardiac muscle by quantifying its deformation during contraction (systolic period) and relaxation (diastolic period). However, these strain measurements suffer from inaccuracies caused by the degradation of the tags and the image quality. In this work, numerical simulations are used to investigate the factors contributing to the error in measurements. An empirical model for the estimated strain values is deduced and presented. In addition, a correction method for the measurement errors is proposed based on the empirically-deduced model. The method was validated on real data, and showed potential enhancement by reducing the errors in strain measurements.
\end{abstract}

Keywords: Strain, Left ventricle regional function, tagged MR.

\section{Introduction}

Coronary artery disease is the leading cause of death worldwide. Blockages in the coronary artery result in the development of ischemic regions in the myocardium those with a reduced flow of blood and oxygen. If this condition persists, these regions may be further stressed causing angina or lead to infarction, resulting in tissue death or necrosis due to loss of blood flow. Depending on the extent of the infarction, a cardiac arrest might occur or sever compensation might lead to heart failure. Accurate quantification and timing of regional myocardial function allows early identification of dysfunction, and becoming increasingly important for clinical risk assessment, patient management, and evaluation of therapeutic efficacy [9].

Strain analysis allows a direct assessment of the degree of regional myocardial deformation and its timing during the cardiac cycle. Ischemic and infarcted regions have different motion patterns than normal regions, which will yield to different strain signals [1]. Tissue strain at any time is percentage change per unit length in some direction [2]. Due to the doughnut shape of the short axis cross section of the heart, strain is usually estimated in two main directions: radial and circumferential. However, myocardial contraction is principally circumferential [9]. The estimated strain signals (strain vs. time curves) are not only used to determine the local deformation of 
myocardium but also can reveal subtle, but important, features relevant to a patient's heart condition [3].

Cardiac Magnetic Resonance (CMR) tagging is considered the gold standard technique for measuring the left ventricular strain [4]. In CMR tagging, non-invasive markers (tag lines) are virtually implanted that move with the motion of heart muscle, Fig.1. Tracking the moving tag lines with time allows quantification of its mechanical which provides a quantitative analysis for the regional function of the heart. For automatic quantification of strain, the Harmonic Phase (HARP) technique automates the process of tracking points inside the heart from tagged MR images [5, 8]. It is based on the idea that the harmonic phase of a point is invariant with time. This means that tracking points implies finding the points that have the same harmonic phase values throughout the entire image sequence. After finding these points, it is easy to just compute the mechanical strain in the two directions; circumferential and radial. Fig.2-a shows an ideal circumferential strain signal covering the cardiac cycle starting from first time frame till $250 \mathrm{msec}$ to cover the systolic period and while rest of the signal covers the diastolic cycle. Its frequency response is shown in Fig.2-b.

Unfortunately, the strain signals estimated from the HARP technique can be distorted, resulting a strain signals such as in Fig.2-c\&d, due to a number of factors that depend on the image quality and acquisition. Factors such as image intrinsic noise, fading of the tags, too small tag separation, and cardiac twisting, have been stated in literature $[5,6]$ but they have not been fully studied to investigate their effect on the strain signal. Moreover, little work has been done to propose remedies for these artifacts.

In this work, the effect of each factor is studied using numerical simulation to determine the extent of distortion induced in the strain signals. An empiricallydeduced model is proposed based on the findings of this study. In addition, a correction method is proposed for the strain signal. The proposed method has been validated on real strain signals and the results show great potential to remove the artifacts from the strain signal.

\section{Theory and Methods}

\subsection{Study of Inherent Causes of Artifacts}

Numerical simulations were conducted to study the extent of corruption caused by the factors stated above. In these simulations, tagged MR image sequences are generated for doughnut-shaped phantom having Longitudinal Relaxation Time $\left(T_{1}=850 \mathrm{msec}\right)$ and inner and outer radii of $30 \mathrm{~mm}$ and $70 \mathrm{~mm}$ respectively. The tag patterns are generated by simulating the Spatial Modulation of Magnetization pulse sequence (SPAMM) [7]. This is achieved by multiplying a uniform-intensity image, $\operatorname{Im}_{0}$, with a finite cosine series (Eq.1) whose frequency corresponds to (1/Tag Separation).

$$
\operatorname{Im}_{\text {tag }}(x, y)=\frac{1}{2} \operatorname{Im}_{0}(x, y)\left(1+\cos \left(\frac{2 \pi}{T S}\right)\right)
$$

where $I m_{\text {tag }}$ is image after imposing the tag patterns with tag separation $T S$. 


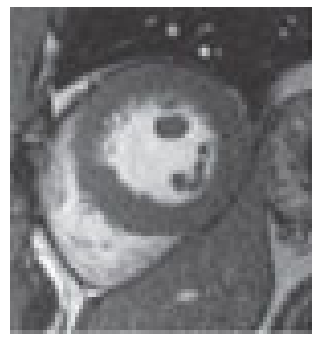

(a)

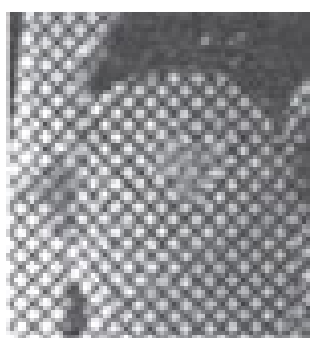

(b)

Fig. 1. (a) MRI image of the Left Ventricle captured during Systolic Period, where (b) shows the corresponding Tagged MR image

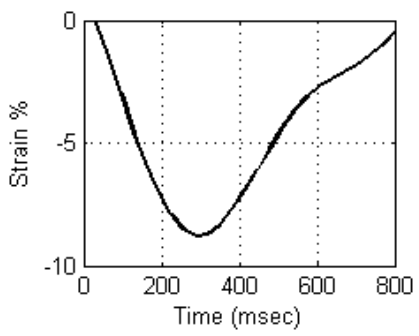

(a)

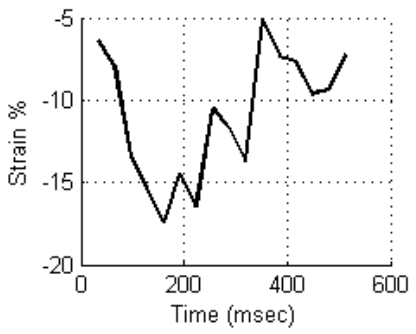

(c)

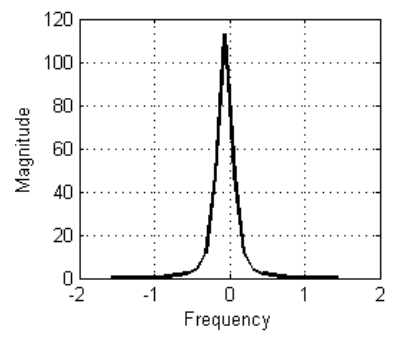

(b)

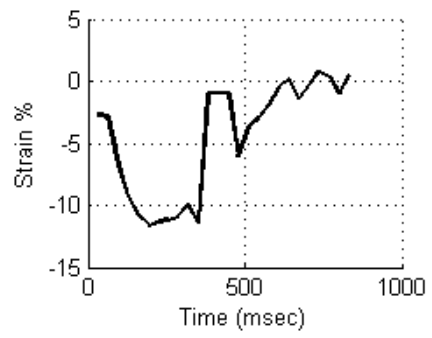

(d)

Fig. 2. (a) Smooth real strain signal (b) The Fourier domain spectrum corresponding to strain signal in (a). (c) \& (d) distorted real strain signals

After generating the tagged MR images shown in Fig.3, simulation of the cardiac motion is done using a predefined function, similar to the signal in Fig.2-a, having only DC and 2 frequency components. The reason for choosing such function will be discussed below. A sample images and strain signals generated by the simulator are shown in Fig.3, 4 respectively.

The next step is the simulation of the factors causing distortion of the strain signal. This is done as follows:

Tag pattern fading depends on the $T_{1}$ relaxation time, $T_{1}=850 \mathrm{msec}$ for the cardiac muscle, as well as the flip angle $(\alpha)$ of the RF pulse sequence [5]. Fading appears in the tagged MR images, as shown in Fig.3-c, as reduction in the tag contrast, or the 
peak-to-peak magnitude of the finite cosine series (Eq.2), which decreases exponentially with increasing $\alpha$ of the RF pulse, that is,

$$
\operatorname{Im}_{\text {tag }}(x, y)=\frac{1}{2} \operatorname{Im}_{0}(x, y)\left[\left(1-e^{-t / T_{1}^{*}}\right)+\left(e^{-t / T_{1}^{*}}\right) \cos \left(\frac{2 \pi}{T S}\right)\right]
$$

where $T_{1}^{*}$ is given as follows;

$$
T_{1}^{*}=\frac{1}{T_{1}}-\frac{\log (\cos \alpha)}{T_{r}}
$$

where $\alpha$ is the RF pulse sequence flip angle and $T_{r}$ is the time to repetition of the pulse sequence.

Small tag separation is simulated by assigning the tag separation ranging from $1 \mathrm{~mm}$ to $6 \mathrm{~mm}$.

Twisting of the heart muscle is simulated by rotating the inner and outer radii of the doughnut-shape centered in the tagged MR image by an angle $\theta$ that varies with time.

Additive intrinsic Noise is simulated by adding white Gaussian noise on the complex image with zero mean and different variance values to the images.

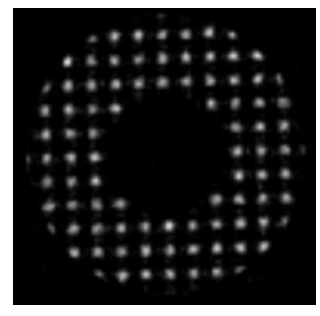

(a)

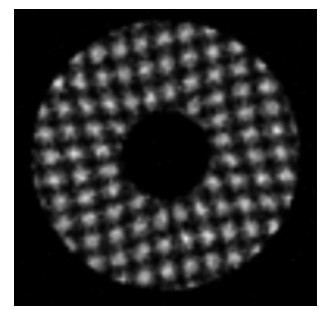

(b)

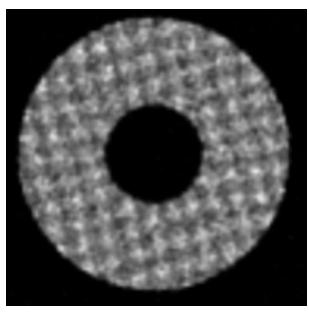

(c)

Fig. 3. Simulated tagged MR image with tag separation $=6 \mathrm{~mm}, \alpha=12^{\circ}$, twisting angle $=30^{\circ}$ and noise variance $=0.005$ captured at (a) 1 st time frame, (b) end-systole, (c) middle diastole.

\subsection{Strain Curve Modeling}

The aim of modeling the strain signal is to extract the strain patterns regardless of the corruption found in the strain signal. Because of the smoothness of the normal strain signals, it was natural to use only the low frequency coefficients of the Fourier series of the strain signal to represent it. In this study, to determine the number of frequency coefficients sufficient to replicate the strain signal, the energy density distribution was calculated from strain signal dataset containing 140 smooth strain signals, i.e. strain signals with minimal artifacts, of normal volunteers and patients. It showed that the first 5 Fourier coefficients, represented in Eq4, contain $98.97 \%$ of the total energy with variance $\pm 0.52 \%$. Moreover, adding higher order of coefficient not only has a minimal effect on extracting the patterns, but it also causes distortion in the strain signal in case of corrupted strain signal. 


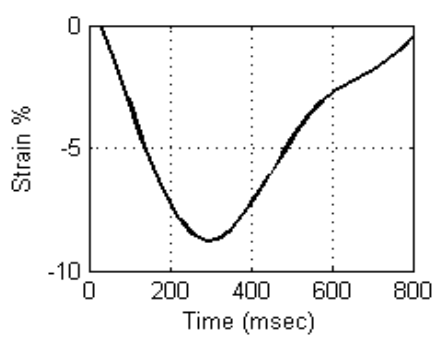

(a)

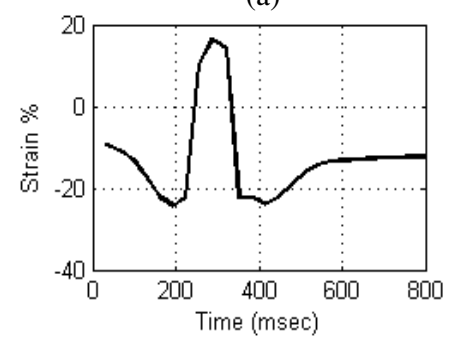

(c)

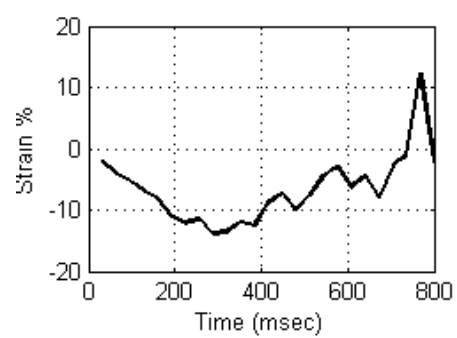

(b)

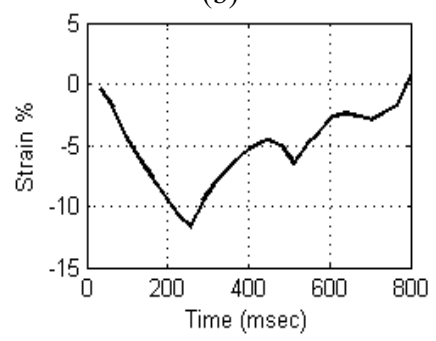

(d)

Fig. 4. Simulated (a) ideal strain signal (b) at noise variance $=0.005$ and $\alpha=12^{\circ}$ (c) at tag separation $=2 \mathrm{~mm}$, (d) at twisting angle $=30^{\circ}$

This model was compared with an ideal low pass filter (LPF) with cut-off frequency equal to the first 5 Fourier coefficients. It was also compared with polynomials of $2^{\text {nd }}$ to $7^{\text {th }}$ order.

$$
\varepsilon_{f i t}(t)=a_{0}+a_{1} \cos (w t)+b_{1} \sin (w t)+a_{2} \cos (2 w t)+b_{2} \sin (2 w t)
$$

where $a_{0}, a_{1}, a_{2}, b_{1}, b_{2}$, and $w$ are the model parameters, $\varepsilon_{f i t}$ is the model fitted on the strain signal.

\subsection{Weighted Least Square Curve Fitting}

Although least square error (LSE) method can be used to fit the above mentioned model to the raw strain signals, instead weighted least-mean squares (LMS) method is used. This allows the use of priori information about our confidence in each strain measurement; i.e. assign error probability for each point. In fact, the inherent causes of the strain artifacts can be dominant in some particular region of the signal.

Tag pattern fading: According to Eq. 2, the tag pattern fades exponentially with time, depending mainly on the flip angle $(\alpha)$ of the RF pulse sequence, since $T_{l}$ is constant in all regions of the cardiac muscle. Therefore, weights can be assigned as the inverse function of the exponential decaying function of the tag fading (Eq.2).

$$
W_{1}(t)=e^{t / T_{1}^{*}}
$$

where $W_{l}$ is the adjusted weights to reduce the fading effect, $T_{1}^{*}$ is defined in Eq.3.

Factor of small tag separation and maximum twisting: It was observed, from simulation, that this factor causes positive peaks to be present in the middle of the 
strain signal, i.e. at peak systole. Therefore, weights are assigned as Gaussian distribution (Eq.6) with mean equal to the time index of peak strain (end-systole) and variance corresponding to region starting from $25 \%$ to $55 \%$ of the strain signal.

$$
W_{2}(t)=\frac{1}{\sqrt{2 \pi \sigma^{2}}} e^{-\frac{(t-\mu)}{2 \sigma^{2}}}
$$

where $W_{2}$ is the adjusted weights to reduce the small tag separation and twisting effect, $\mu$ is the time index of peak strain and $\sigma$ is the variance.

Additive intrinsic noise: Due to the randomness of its source, we assumed uniform weights at each strain sample. Hence, combining all these information together, we get overall weights along the strain signal (Fig.5). The weights are adjusted so that maximum weight magnitude corresponds to highest confidence.

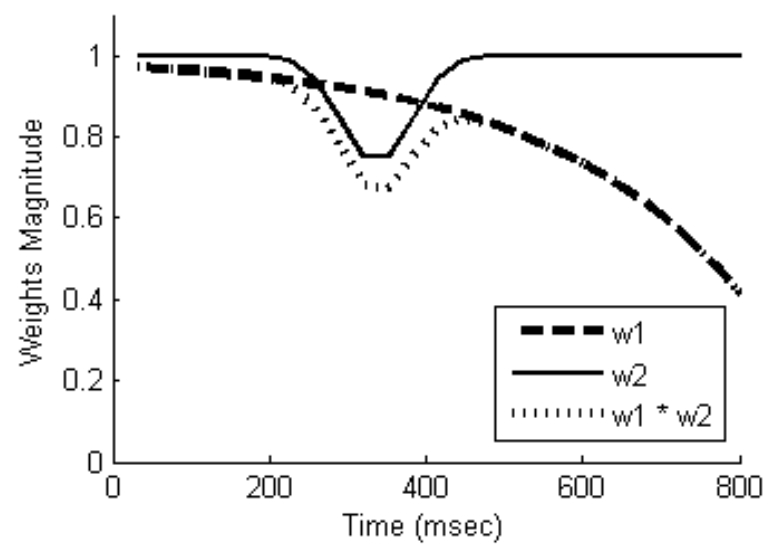

Fig. 5. The weights of the strain curve fitting problem

\section{Results}

A dataset, acquired from the MESA study containing 840 strain signals for 70 volunteers and patients, is used to evaluate the proposed method. For each subject 12 strain signals were acquired corresponding to different segment of the cardiac muscle.

A comparison, based on computing error using Eq.7 and its variance, is done on 140 smoothed strain signals, i.e. with minimal artifacts, between the model presented in this study, and an ideal LPF with cut-off frequency equal to the 5 Fourier coefficients, and also different fitting models such as; $2^{\text {nd }}$ till $7^{\text {th }}$ polynomial. The results of the comparison are shown in Table1.

$$
\text { error }=\sqrt{\sum_{t=0}^{T} \frac{\left(\varepsilon_{\text {fit }}^{2}(t)-\varepsilon_{\text {actual }}^{2}(t)\right)^{2}}{\varepsilon_{\text {actual }}^{2}}} \times 100
$$

Where $\varepsilon_{f i t}$ is the resultant strain signal from the fitting models, $\varepsilon_{\text {actual }}$ is the actual strain signal and $T$ is the time covering the strain signal. 
Table 1. Error percentage computed for different model

\begin{tabular}{c|c|c|}
\hline Fitting Model & Mean & Variance \\
\hline $\mathbf{2}^{\text {nd }}$ poly & $32 \%$ & $11.5 \%$ \\
\hline $\mathbf{3}^{\text {rd }}$ poly & $16 \%$ & $6 \%$ \\
\hline $\mathbf{4}^{\text {th }}$ poly & $13.4 \%$ & $6.4 \%$ \\
\hline $\mathbf{5}^{\text {th }}$ poly & $8.1 \%$ & $3.9 \%$ \\
\hline $\mathbf{6}^{\text {th }}$ poly & $7.2 \%$ & $3.5 \%$ \\
\hline $7^{\text {th }}$ poly & $6.2 \%$ & $2.9 \%$ \\
\hline Low Pass Filter & $11.5 \%$ & $7.1 \%$ \\
\hline $\mathbf{5}$ Fourier Coeff. & $7.3 \%$ & $2.8 \%$ \\
\hline
\end{tabular}

Table 2. Percentage of strain signals which the experts were able to extract information from before and after the proposed method

\begin{tabular}{c|c|c|} 
& Before & After \\
\hline Strain signal & $21.014 \%$ & $80.43 \%$ \\
\hline Systolic Period & $49.8 \%$ & $83.6 \%$ \\
\hline Diastolic Period & $22.5 \%$ & $80.2 \%$ \\
\hline
\end{tabular}

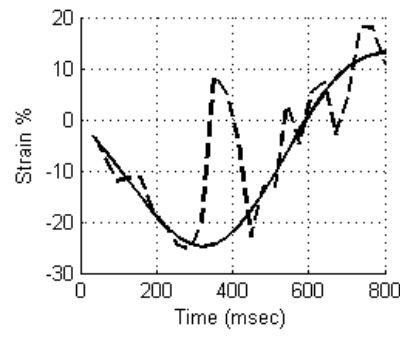

(a)

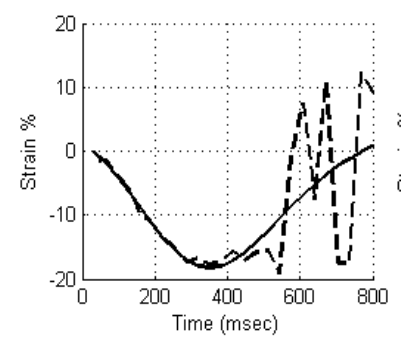

(d)

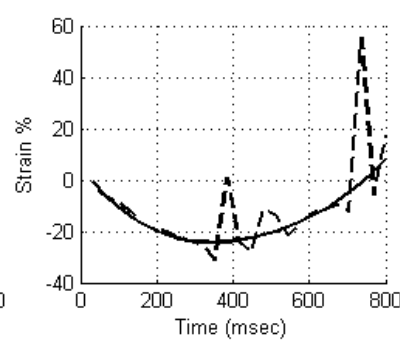

(b)

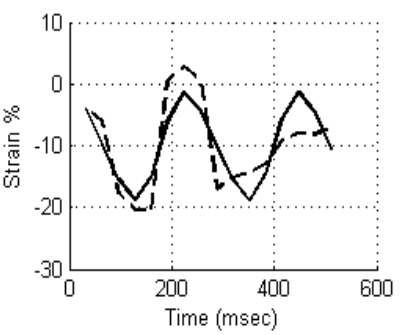

(e)

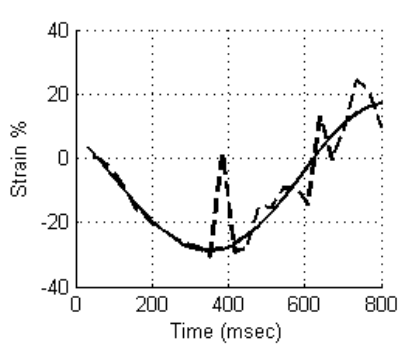

(c)

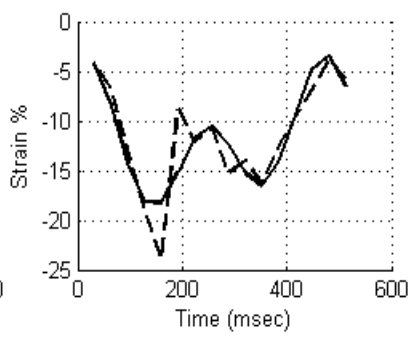

(f)

Fig. 6. Success and failure in 6 real strain signals before (dotted) and after (solid) applying the proposed method. (a)-(d) successful correction while (e)\&(f) failure in correcting strain signals.

In case of distorted strain signals, the performance of the proposed method is evaluated visually by experts in the field of CMR. This evaluation cannot be done 
automatically due to the lack of a gold reference for the strain signals. Experts were conducting a binary evaluation on the strain signals, i.e. they assign zero to the strain signals from which they could not extract useful information. They have evaluated the entire strain signal as well as the parts covering the systolic and diastolic periods only. Experts' evaluation results are shown in Table2. Success and failure samples are shown in Fig.6.

\section{Discussion}

Simulations done in the study provided good understanding of the inherent causes of artifacts and how these artifacts distort the strain signal. Therefore, it was found that artifacts resulting from mistracking of the tag lines due to fading of the tag patterns increases with the decrease of the image quality and the increase in the RF flip angle. As for small tag separation, their artifacts appears as positive spikes, as shown in Fig.4-c, in the region of end-systole of the strain signal due to the fact that at maximum contraction the small tag lines overlap making some tag lines disappear. Twisting of the heart muscle showed artifacts quite similar to the one coming from small tag separations, it also appears as positive spikes but found in larger region in the strain curves as shown in Fig.4-d.

Ideal LPF was expected to provide mean error similar or better comparable with the other models, but Table1 shows that applying it on smoothed strain signals, i.e. with minimal artifacts, has mean error of $11.5 \%$ which is higher than the model proposed in the study. Moreover, in case of distorted strain signals, its results were biased by the severity of artifacts found in the signal. Therefore, using an ideal LPF in this case would get poor improvement in the results.

Modeling the strain signal with polynomials of different orders showed that by increasing the order of polynomials the mean error decreases. The $6^{\text {th }}$ and $7^{\text {th }}$ polynomial has mean error comparable with the proposed model, but they suffered from over fitting when tested on real distorted strain signals.

Based on the energy density distribution of strain signals, it was found that the first 5 Fourier coefficients stores energy of $98.97 \%$ with variance equal to $\pm 0.52 \%$ of the total energy. Therefore, based on this finding, it can be said that the proposed model is sufficient to replicate the strain signal regardless of the patient's condition.

Table 2 shows that the efficiency of extracting information from the strain signals has increased up to $80 \%$ instead of $20 \%$ after applying the weighted LMS fitting method. It showed a good potential in reducing the artifacts caused by the fading effect at low signal-to-noise ratio as well the small tag separation and twisting of the heart muscle. The remaining $20 \%$ of the strain signals needs further analysis to understand more the inherent causes of artifacts in order to increase the efficiency.

\section{Conclusion}

In this work, the extent of induced distortion in the strain signals is studied using numerical simulation. Based on the energy density distribution from dataset used in this study, an empirically-deduced model is presented. This model is used for the 
correction of distorted strain signals using weighted LMS method. Evaluation of the proposed method was done on real strain signals by experts in the field. It showed good potential in enhancing the strain signals.

\section{References}

1. Fahmy, A.S., Krieger, A., Osman, N.F.: An integrated System for Real-Time Detection of Stiff Masses with a Single Compression. IEEE Transaction on Biomedical Engineering 53(7) (July 2006)

2. Urheim, S., Edvardsen, T., Torp, H., Angelsen, B., Smiseth, O.A.: Myocardial Strain by Doppler Echocardiography: Validation of a new Method to quantify regional myocardial function. Circulation 102, 1158-1164 (2000)

3. Stuber, M., Scheidegger, Fischer, S.E., Nagel, E., Steinenmann, F., Hess, O.M., Boesiger, P.: Alterations in the local myocardial motion pattern in patients suffering from pressure overload due to aortic stenosis. Circulation 100, 361-368 (1999)

4. Youssef, A., Ibrahim, E.-S.H., Korosoglou, G., Abraham, R., Weiss, R.G., Osman, N.F.: Strain-Encoding cardiovascular magnetic resonance for assessment of right-ventricular regional function. Journal of Cardiovascular Magnetic Resonance 10, 33 (2008)

5. Osman, N.F.: Measuring Regional Cardiac Function Using Harmonic Phase Magnetic Resonance Imaging. A dissertation submitted to the Johns Hopkins University in conformity with the requirements for the degree of Doctor of Philosophy (May 2001)

6. Parthasarathy, V.: Characterization of Harmonic Phase MRI: Theory, Simulation and Applications. A dissertation submitted to the Johns Hopkins University in conformity with the requirements for the degree of Doctor of Philosophy (April 2006)

7. Axel, L., et al.: MR imaging of motion with spatial modulation of magnetization. Radiology 171, 841-845 (1989)

8. Osman, N.F., Kerwin, W.S., McVeigh, E.R., Prince, J.L.: Cardiac Motion Tracking Using CINE Harmonic Phase (HARP) Magnetic Resonance Imaging. Magnetic Resonance in Medicine 42, 1048-1060 (1999)

9. Götte, M.J.W., et al.: Myocardial Strain and Torsion Quantified by Cardiovascular Magnetic Resonance Tissue Tagging: Studies in Normal and Impaired Left Ventricle. Journal of the American College of Cardiology 48(10) 